Musées, Patrimoine et Culture scientifiques et techniques

$186 \mid 2019$

novembre-décembre 2019

\title{
Trous noirs. De l'image scientifique à l'image de médiation
}

Marion Parisis

\section{OpenEdition}

Journals

Édition électronique

URL : http://journals.openedition.org/ocim/3432

DOI : 10.4000/ocim.3432

ISSN : 2108-646X

Éditeur

OCIM

Édition imprimée

Date de publication : 1 novembre 2019

Pagination : 29-35

ISSN : 0994-1908

Référence électronique

Marion Parisis, «Trous noirs. De l'image scientifique à l'image de médiation », La Lettre de l'OCIM [En ligne], 186 | 2019, mis en ligne le 01 novembre 2020, consulté le 25 janvier 2021. URL : http:// journals.openedition.org/ocim/3432 ; DOI : https://doi.org/10.4000/ocim.3432

Ce document a été généré automatiquement le 25 janvier 2021.

Tous droits réservés 


\title{
Trous noirs. De l'image scientifique à l'image de médiation
}

\author{
Marion Parisis
}

L'auteur adresse ses remerciements à Éric Triquet, professeur des universités en Sciences de l'Information et de la Communication (Centre Norbert Elias, université d'Avignon), qui a dirigé son mémoire de recherche et à Daniel Jacobi, professeur émérite (Centre Norbert Elias, université d'Avignon), pour son accompagnement et ses conseils dans la rédaction de l'article. 
Image scientifique du rayonnement émis par un trou noir supermassif (CXO J101527.2+625911) détecté par le téléscope en orbite Chandra (Nasa). Les émissions de rayons $X$ sont issues du disque de matière chaude qui se forme lorsqu'un trou noir accrète de la matière.

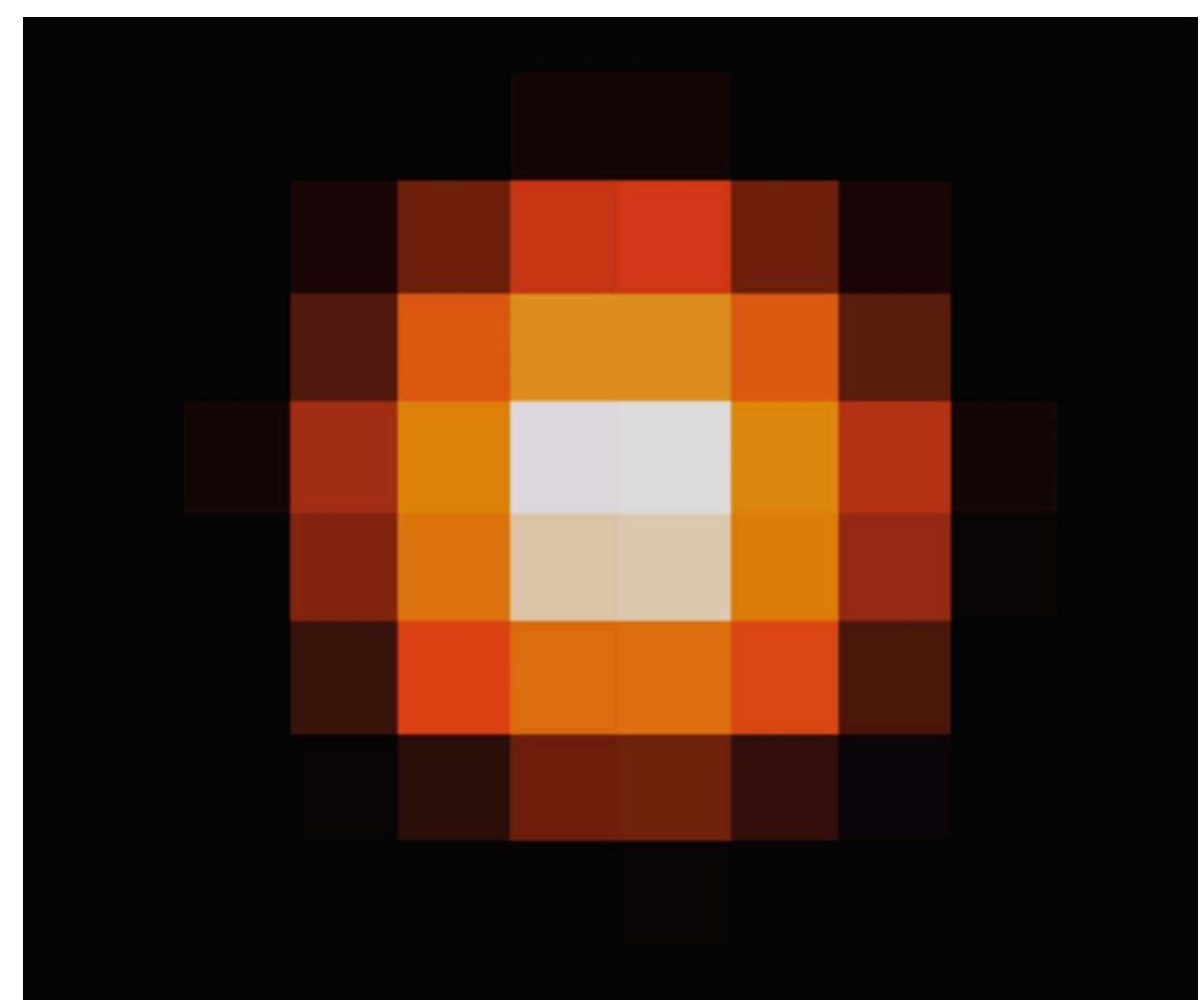

(c) Nasa/CXC/NRAO/D.-C.Kim,2017

1 Les trous noirs font partie des objets astrophysiques les plus étudiés de la science et plébiscités par les publics. En effet, au cours de pratiquement chaque visite guidée ou séance de planétarium, le public interroge le médiateur sur ces objets : "Qu'est-ce qu'un trou noir? ", "Que se passe-t-il à l'intérieur? ", " Est-ce vrai que le temps ralentit près d'un trou noir?». Ces questionnements montrent l'intérêt et la curiosité qui s'expriment pour ces objets astrophysiques. On comprendra donc qu'en dépit de la complexité du thème, de nombreuses institutions de culture scientifique abordent la thématique des trous noirs dans leurs animations ou expositions. Parmi les questions spontanées des visiteurs, l'une d'entre elles a particulièrement attiré notre attention: "À quoi ça ressemble puisque c'est noir?». En effet, les médiateurs ont recours à une grande variété d'images. Cependant, une difficulté surgit d'emblée : rien ne peut échapper aux trous noirs, pas même la lumière (voir encadré page suivante). Or, si aucune source de lumière en émane, comment représenter un objet qui est par définition invisible ? Les médiateurs et les institutions de diffusion de la recherche en astrophysique disposent bien sûr d'images scientifiques publiées par des astrophysiciens mais qui ne sont pas forcément celles diffusées auprès des publics non-spécialistes. Nous allons ainsi dégager les différences entre représentations scientifiques et images de médiation et revenir sur le rôle de la recherche scientifique dans les images produites.

\section{Comprendre le trou noir}

Une définition 
Un trou noir est un objet extrêmement massif qui possède un champ gravitationnel intense. Si l'on voulait se mettre en orbite autour d'un trou noir, il faudrait une énergie considérable pour ne pas tomber à l'intérieur (Riazuelo, 2016). Cette gravité est tellement grande qu'elle courbe à l'infini le tissu d'espacetemps situé autour du trou noir. La lumière qui suit l'espace-temps, peut se trouver piégée à l'intérieur. La zone à partir de laquelle la lumière est définitivement piégée se nomme l'horizon des événements. Si aucune lumière ne peut s'en échapper, cette zone est alors naturellement noire. Autour d'un trou noir peut orbiter un disque de matière composé de poussières et de gaz, appelé disque d'accrétion.

Perception de la lumière et des couleurs en astrophysique

Il existe différents types de lumière : la lumière visible, que nos yeux peuvent percevoir, mais aussi celles qui sont imperceptibles pour l'œil humain qui nécessitent un appareillage scientifique pour être mises en évidence. Il est possible d'observer ces différents types de lumière sur des objets célestes, comme des galaxies. Celles-ci font apparaître différentes caractéristiques de l'objet observé. Les courbes sur le schéma s'appellent des longueurs d'onde. Plus celle-ci est courte, plus la lumière est dite énergétique et vire au bleu, plus elle est longue moins elle transporte de l'énergie et vire au rouge foncé. La lumière qui est piégée dans un trou noir perd son énergie et ne libère donc plus de couleur vers l'extérieur de celui-ci.

Schéma sur la perception des lumières.

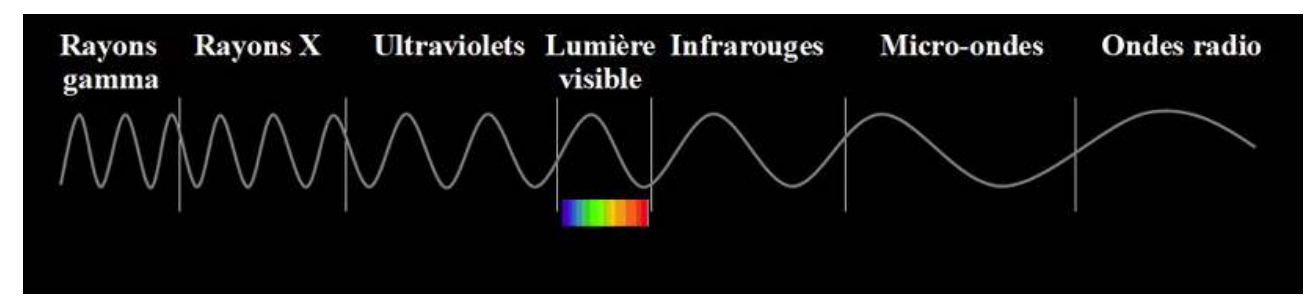

\section{La production des images scientifiques de trous noirs}

\section{Des images produites à partir de modèles théoriques}

Deux représentations scientifiques de trous noirs servent principalement de référence. En 1979, Jean-Pierre Luminet, astrophysicien, a publié la première image de la forme apparente d'un trou noir entouré d'un disque d'accrétion (figure 1). Celle-ci est en noir et blanc et représente la forme qu'aurait un trou noir si l'on pouvait l'observer. En 1995, l'astrophysicien Jean-Alain Marck, a produit la même image mais actualisée, en couleurs et sous la forme d'une vidéo (figure 2). Le disque d'accrétion d'un trou noir s'illumine puisqu'il est très chaud. Les auteurs ont donc utilisé la présence d'un tel disque pour faire ressortir la silhouette du trou noir. Ces images ont été produites à partir de notions théoriques modélisées informatiquement. 
Figure 1 : Représentation d'un trou noir par l'astrophysicien Jean-Pierre Luminet en 1979 (Image of a spherical black hole with thin accretion disk, Astronomy \& Astrophysics, ${ }^{\circ} 75, \mathrm{pp} .228-235$ ).

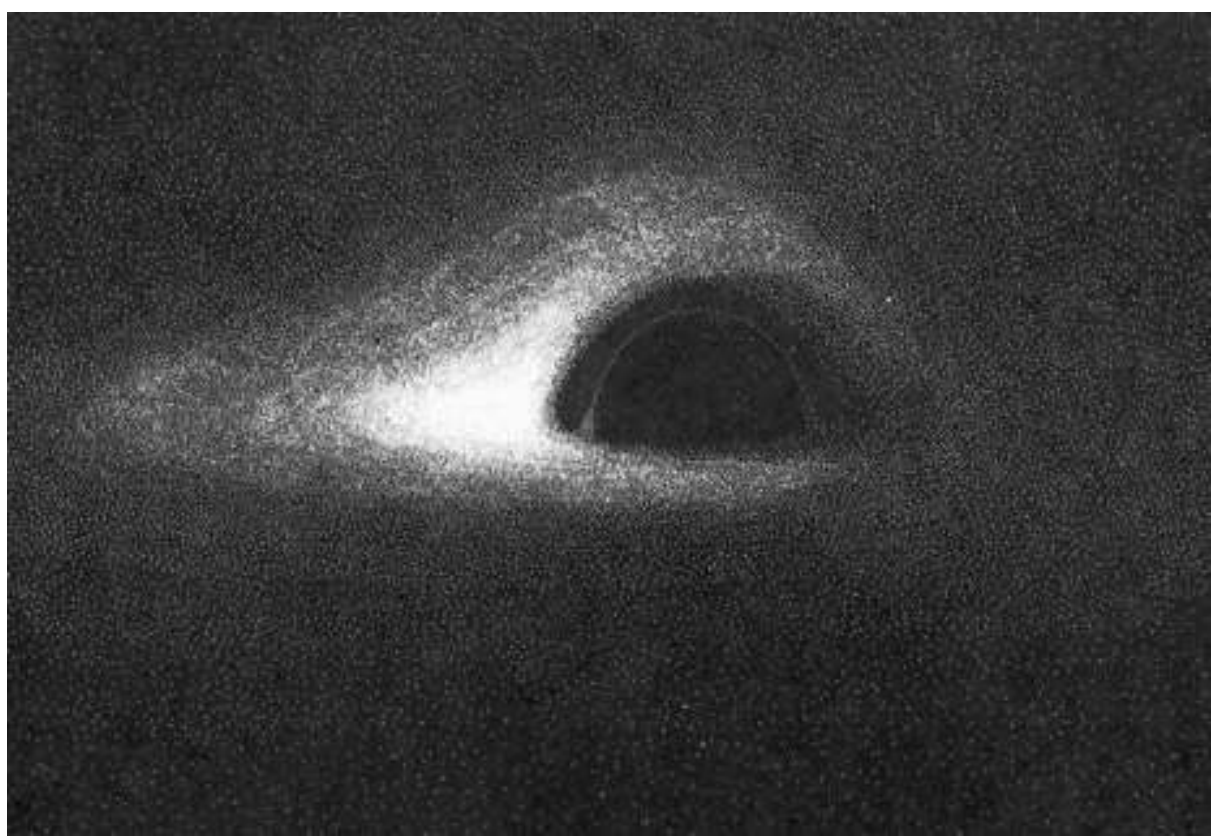

(C) J.-P. Luminet, 1979

Figure 2 : Représentation d'un trou noir par l'astrophysicien Jean-Alain Marck (Shortcut method of solutions of geodesic equations for schwarzschild black hole, Classical and Quantum gravity, Vol. 13 , n³, 1996, pp. 1-16).

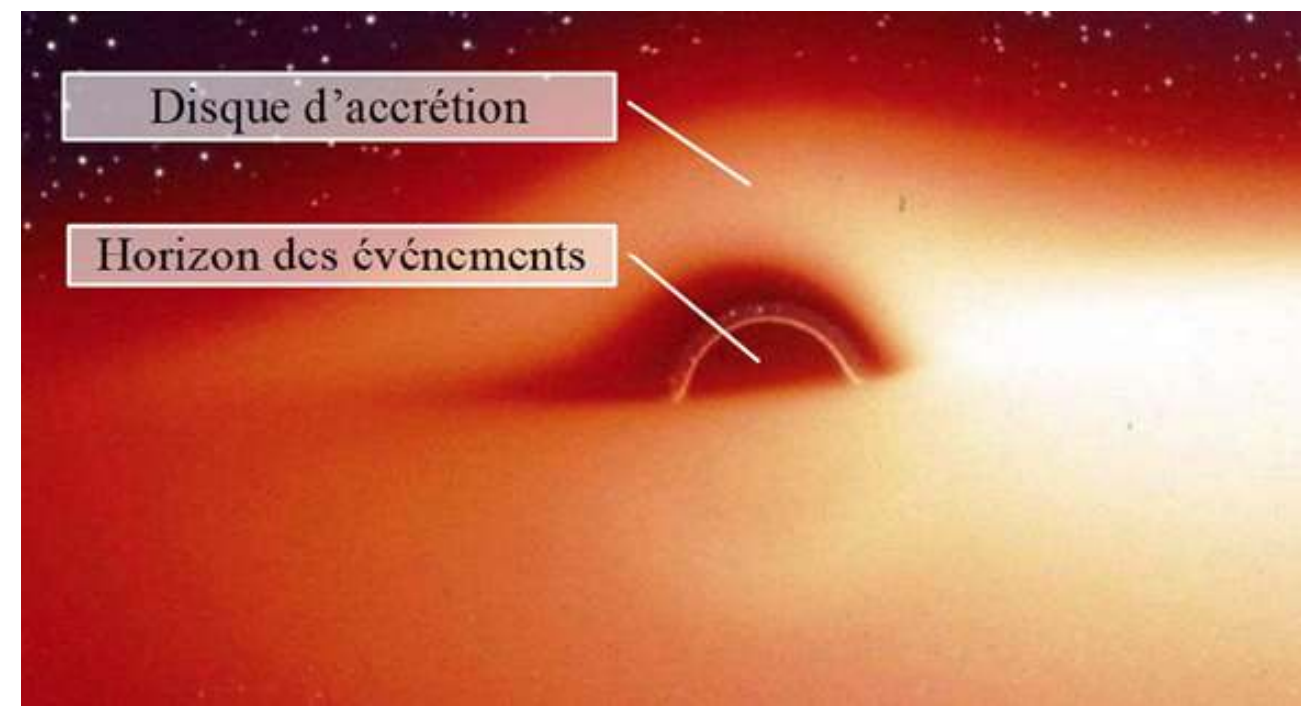

(C) Jean-Alain Marck, 1995, légendé par Marion Parisis 
Figure 3 : Schéma représentant la déviation des rayons lumineux par la présence d'un trou noir.

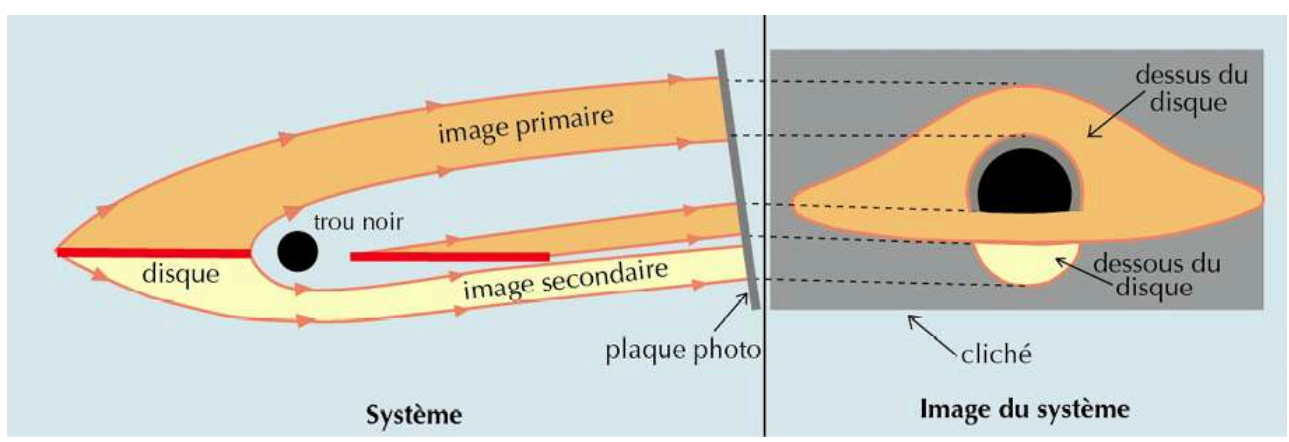

(c) Jean-Pierre Luminet, 2006

3 Sur les deux images on remarque que le disque d'accrétion semble passer au-dessus de l'horizon des événements et qu'il y a un dégradé de gauche à droite. Explicitons ces effets visuels. D'abord, les objets massifs ont la particularité de dévier la trajectoire des rayons lumineux. Puisque qu'un trou noir est un objet particulièrement massif, ce dernier peut dévier la lumière qui émane de son propre disque d'accrétion. Ainsi, la lumière du disque arrière est déviée et se retrouve au-dessus de l'horizon du trou noir (figure 3), à la manière d'un chapeau (Luminet, 2006).

4 Le dégradé de gauche à droite (figures 1 et 2) est causé par l'effet Doppler. Ce dernier se manifeste par exemple lorsqu'un camion de pompier passe à côté de nous. En s'approchant, la sirène du camion est aiguë, et lorsqu'il s'éloigne le son devient de plus en plus grave. Il existe le même phénomène avec la lumière qui émane du disque d'accrétion. Lorsque le disque s'approche d'un observateur sa lumière tend vers le bleu, et lorsqu'il s'éloigne elle tend vers le rouge (Luminet, 2006) ${ }^{1}$. Ce dégradé de couleur nous informe sur le sens de rotation du disque. Enfin, dans les images précitées, on remarque une partie sombre au niveau du disque d'accrétion intérieur, c'est-à-dire, très proche de l'horizon des événements. En effet, une partie de la lumière est piégée à l'intérieur d'un trou noir, mais une autre partie située à la limite intérieure du disque peut s'en échapper. En s'échappant, cette lumière perd de l'énergie et nous apparait alors plus sombre : c'est ce que l'on appelle l'effet gravitationnel.

\section{Des images reconstruites informatiquementà partir de détections}

5 Très récemment, le 10 avril 2019, le réseau Event Horizon Telescope a diffusé la première "photographie» d'un trou noir. Cette collaboration entre une dizaine de radiotélescopes répartis sur la surface du globe ${ }^{2}$ avait pour objectif d'observer pour la première fois un trou noir, et ainsi tenter de confirmer la forme apparente d'un tel objet, comme l'avait travaillé J.-P. Luminet (figure 1). Malgré ce que les publics ont pu lire dans les médias, il ne s'agit pas d'une photographie au sens commun du terme. En réalité, cette image (figure 4) a été reconstruite informatiquement à partir d'une série de détections faibles et éparses de la lumière qui émane du disque d'accrétion autour du trou noir ${ }^{3}$. Des calculs informatiques ont été développés pour transformer ces détections, initialement chiffrées et graphiques, en image ${ }^{4}$.

Sur la figure 4 , on remarque un dégradé du blanc au rouge qui semble témoigner de l'effet Doppler. Cependant, il ne s'agit pas des couleurs naturelles détectées. Les couleurs ont été « rajoutées » par les scientifiques pour faire ressortir les effets subis 
par le disque d'accrétion autour du trou noir ${ }^{5}$. Ensuite, comme pour les figures 1 et 2 on peut voir que le disque d'accrétion semble passer au-dessus de l'horizon des événements comme un chapeau. Enfin, la zone noire au centre semble témoigner de la présence d'un horizon 6 .

7 Cependant, nous ne pouvons pas encore présenter cette image scientifique en tant que preuve irréfutable de l'existence des trous noirs, ni même affirmer qu'il s'agit bien de l'apparence de tels objets. Même si ces résultats sont encourageants, en raison de la nature rare et éparse des données récoltées, il demeure des doutes sur la nature de l'objet détecté, celui-ci même qui suggère les effets subis par le disque d'accrétion autour de lui ${ }^{7}$. Ces éléments suggèrent la nature hypothétique des images scientifiques de trous noirs, voire même des trous noirs eux-mêmes. Grâce aux futures détections, il sera possible de préciser la nature de l'objet, mais aussi son apparence (figure 4$)^{8}$.

Figure 4 : Reconstitution de l'image du trou noir supermassif situé au centre de la galaxie nommée M87.

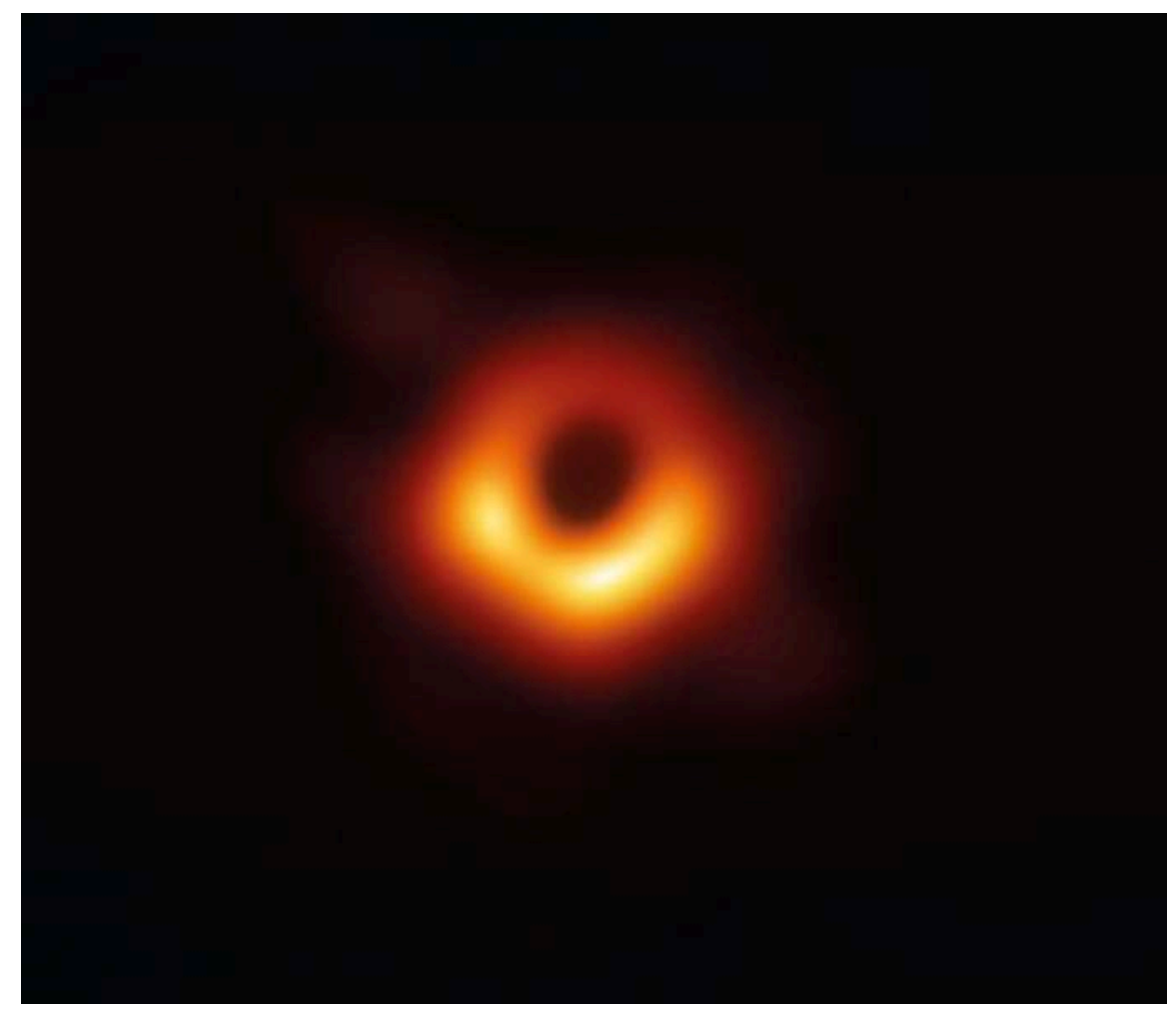

(c) Collaboration Event Horizon Telescope, 2019

\section{Les représentations vulgarisées des trous noirs: exemple de deux images}

Dans le champ de la médiation, les images diffusées semblent plus ou moins différentes, non seulement entre elles, mais aussi par rapport à celles produites par les scientifiques. La plupart des images issues de la médiation montrent des trous noirs possédant un disque d'accrétion plat avec un dégradé de couleur allant du marron/ rouge au blanc, du disque externe vers le disque interne (figure 5 et 6). Pour étudier les différences entre les images issues du cercle scientifique (figures 1,2 et 4 ) et celles qui 
sont diffusées auprès des publics (figures 5 et 6), nous avons réalisé une étude de cas sur les représentations visuelles de trous noirs de l'ESO et de la Nasa ${ }^{10}$. Ces deux images peuvent être diffusées au sein de structures comme des planétariums ou à l'occasion d'expositions ${ }^{11}$.

Figure 5 : Images de trous noirs utilisées en médiation et présentant un disque plat d'accrétion.

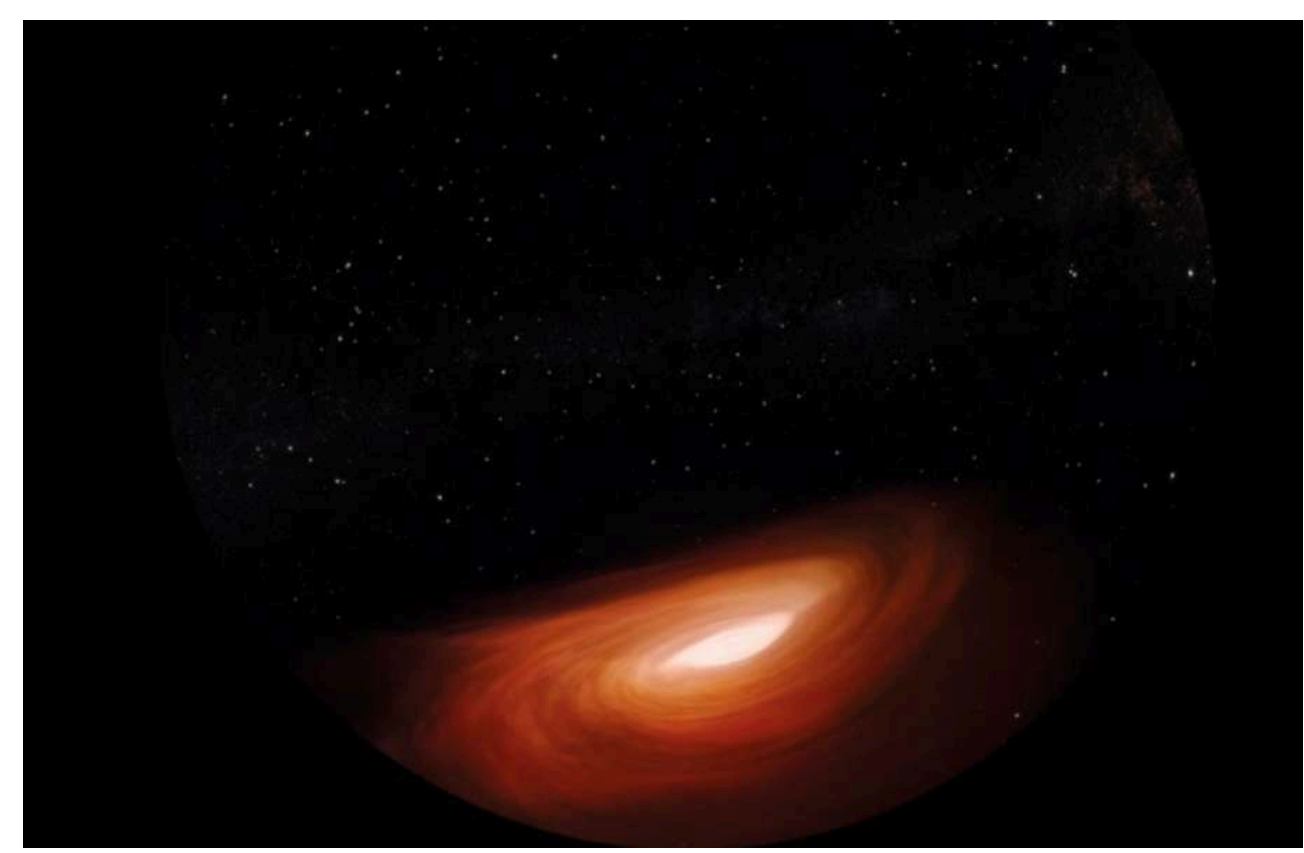

(c) ESO/L.Calcada/M.Kornmesser, 2014

Figure 6 : Images de trous noirs utilisées en médiation et présentant un disque plat d'accrétion.

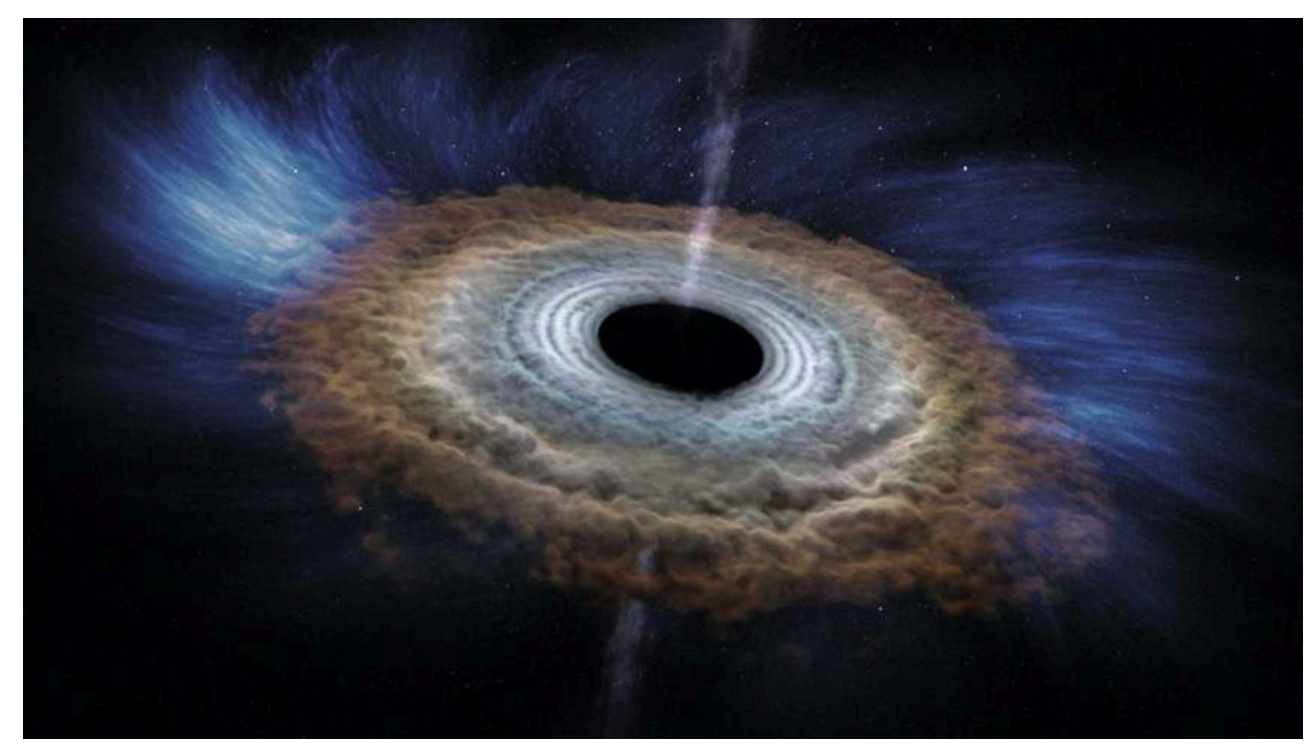

(C) Nasa's Goddard Space Flight Center/CI Lab, 2016

9 Sur les figures 5 et 6 , le disque d'accrétion est représenté plat, alors que sur les images de Luminet et Marck (figures 1 et 2), celui-ci est représenté plat devant et courbe audessus. Les représentations des disques d'accrétion de ces images ne considèrent pas la déviation de la lumière par les masses. Cette simplification ne confère pas forcément à 
ces images un caractère non valide. Si l'on ne prend pas en considération cet effet qui donne un aspect déformé aux objets, le disque d'accrétion peut être plat. Ce disque tourne en effet à grande vitesse autour du trou noir, or la vitesse et la rotation ont tendance à aplatir les objets, à la manière d'un pizzaiolo qui fait tourner sa pâte (force centrifuge). Remarquons aussi les couleurs de ces disques. Celles-ci peuvent représenter les variations de température entre le bord externe et le bord interne du disque. Le bord interne, qui tourne plus rapidement que le bord externe, entraîne un échauffement plus élevé de la matière. Ainsi, à mesure que l'on se rapproche du trou noir, la température du disque augmente (Luminet, 2006). De manière générale, plus un objet est chaud, plus sa lumière tend vers le bleu, moins il est chaud, plus celle-ci tend vers le rouge. C'est ce que l'on voit sur les figures 5 et 6 . Mais contrairement aux images de Luminet et Marck, il manque deux notions dans les images de médiation: l'effet Doppler et l'effet gravitationnel.

10 Cependant les figures 5 et 6 ne sont pas totalement dépourvues de notions scientifiques et les informations qu'elles présentent ne sont pas non plus invalides. Mais, celles-ci sont différentes de l'image produite par les scientifiques (figures 1 et 2). Alors que les trous noirs dans la médiation sont représentés avec les notions d'horizon des événements, de force centrifuge et de température du disque d'accrétion, les images scientifiques mettent en avant les effets qui donneraient au trou noir la forme que l'on observerait (effet Doppler, effet gravitationnel, déviation de la lumière). Les images sont donc adaptées aux notions que l'on souhaite faire partager avec les publics.

\section{Deux modes de visualisation des trous noirs}

11 Notre analyse explique en quoi les images de médiation sont différentes des images produites par les chercheurs, notamment au niveau du contenu scientifique présenté. Il est donc légitime de se demander si les images de médiation dénaturent (ou ne dénaturent pas) l'objet trou noir.

Certes, les images de médiation ne représentent pas certains effets inhérents à sa présence, mais l'objet trou noir en tant que tel n'est pas dépourvu de certaines caractéristiques comme la présence d'un horizon des événements ou d'un disque d'accrétion. Cette étude conduit à distinguer deux catégories d'images pour visualiser les trous noirs : l'image de la forme apparente et l'image de la forme absolue.

Pour désigner ces deux catégories, nous employons le lexique astronomique, à savoir : magnitude apparente (luminosité d'un objet vu depuis la Terre) et magnitude absolue (luminosité intrinsèque de ce même objet). Ainsi, nous considérons la forme apparente d'un trou noir à la manière des astrophysiciens comme Luminet (figure 1), c'est-à-dire, à quoi ressemblerait un trou noir si on l'observait. L'image de la forme apparente comprend tous les effets qui se manifestent de manière visuelle dans le voisinage plus ou moins proche des trous noirs. Pour les images de médiation (figures 4 et 5), on voit que les représentations de trous noirs perdent certains de ces effets visuels au niveau du disque d'accrétion: déviation de la lumière, effet Doppler, effet gravitationnel. Celui-ci est plutôt représenté plat avec des couleurs montrant les variations de température. Ces images sont-elles pour autant non valides? Même si des éléments notionnels sont absents, les caractéristiques de base (horizon des événements et disque d'accrétion) sont présentes. Ces caractéristiques manifestent la forme absolue des trous noirs, c'est-à-dire la forme qu'ils ont de manière intrinsèque si on ne considère pas les 
effets visuels inhérents à ces objets. Ici, on répond à la simple question « quels sont les éléments qui composent un trou noir » dans un contexte de présentation de l'objet.

\section{Relations de la médiation avec les avancées scientifiques}

14 Notre étude débouche sur un thème classique : les représentations visuelles diffusées dans la médiation sont-elles des vues réalistes ou seulement des points de vue? Où se situe l'image réelle entre la forme absolue et la forme apparente des trous noirs? Comment définir la notion de "réalité » dans le domaine de la recherche en astrophysique? Toutes les images scientifiques de trous noirs sont à l'heure actuelle soit le résultat d'hypothèses et de leur mise en équation (figure 1); soit la reconstruction informatisée d'un trou noir à partir de données rares et éparses (figure 4). Lorsque la conception des images de trous noirs demande un effort d'abstraction pour leur conférer une apparence à partir de données mathématiques (Parisis, 2017), elles ne sont dans un sens, pas fidèles à la réalité puisqu'elles ne constituent pas une preuve directe comme les photographies obtenues avec des télescopes comme Hubble par exemple (Parisis, 2017). De plus, quand bien même les scientifiques reconstruisent informatiquement des images de trous noirs à partir d'une faible quantité de détection, cela ne garantit pas une certitude quant à l'apparence de l'objet, ni sa nature (figure 4). En dépit du fait qu'il s'agisse d'une détection directe, les données récoltées sont, pour l'heure, insuffisantes pour confirmer ces éléments ${ }^{12}$.

Néanmoins, ces images de trous noirs, qu'elles soient issues du cercle scientifique ou de la médiation, permettent de projeter les visiteurs dans un monde éventuellement réel que l'on peut qualifier comme une fiction scientifique. Selon David Lewis (2012), la fiction scientifique revient à imaginer des événements en laissant de côté les règles du monde que nous connaissons afin d'en découvrir d'autres. L'auteur compare la fiction scientifique à la construction de mondes contrefactuels. C'est en s'ouvrant au champ des possibles que nous pouvons comprendre le monde et les objets qui peuplent l'univers. En utilisant en partie la fiction scientifique, les astrophysiciens ont imaginé que les trous noirs pouvaient exister. Mais même après avoir obtenu plusieurs preuves de leur existence, des questionnements demeurent : il est possible que les trous noirs existent, mais quelles alternatives sont encore possibles? Autrement dit, les représentations visuelles que l'on attribue aux trous noirs peuvent-elles être applicables à d'autres objets encore non découverts? Ceci peut expliquer le foisonnement d'images et l'intérêt grandissant des astrophysiciens pour obtenir une réponse à ces questions. Peut-être que les prochaines détections de l'Event Horizon Telescope permettront de le préciser et feront évoluer notre analyse. Ce questionnement se pose aussi pour de nombreux autres objets astrophysiques qui font l'objet de recherches (énergie noire, matière noire, planète 9...). Notre travail pose donc la question de la relation de la médiation aux avancées scientifiques : de quelle manière la médiation scientifique va t-elle se saisir de ces actualités? Quoi qu'il en soit, montrer de telles images aux publics non-spécialistes implique une formation des médiateurs, et d'autant plus lorsqu'il s'agit de présenter des images de trous noirs issues du champ scientifique (figures 1,2 et 4 ). 


\section{BIBLIOGRAPHIE}

Lewis, D. La philosophie de David Lewis, Klésis, revue philosophique, n²4, 2012, pp. 36-55.

Luminet, J.-P. Image of a Spherical Black Hole with Thin Accretion Disk, Astronomy and Astrophysics, $\mathrm{n}^{\circ} 75,1979$, pp. 228-235.

Luminet, J.-P. Le destin de l'Univers II. Éditions Gallimard : Paris, 2006, 532 p.

Marck, J.-A. Black holes (The). 1992. Vidéo documentaire du CNRS : https://videotheque.cnrs.fr/ doc $=46$

Parisis, M. La médiation de l'astronomie invisible : le cas des planétariums numériques. Pré-mémoire de Master en Sciences de l'Information et de la Communication. Université d'Avignon et des Pays de Vaucluse, 2017.

Riazuelo, A. et Science \& Avenir. Voyage au cœur d'un trou noir. 2006. Vidéo de 37 minutes : www.youtube.com/watch?v=GWRzVyX53ZU

Riazuelo, A. Les trous noirs, à la poursuite de l'invisible. Paris : Vuibert, 2016, 189 p.

\section{NOTES}

1. Le même phénomène se manifeste pour l'image de Jean-Pierre Luminet mais sous la forme de nuances de gris. En 1979, la technologie ne permettait pas d'ajouter des couleurs.

2. Mettre en réseau plusieurs radiotélescopes permet d'avoir la résolution nécessaire pour détecter l'environnement proche d'un trou noir (eventhorizontelescope.org).

3. Event Horizon Telescope collaboration. First M87 Event Horizon Telescope results IV. Imaging the central supermassive black hole, The Astrophysical Journal Letters, vol. 875, n 1, 2019, $52 \mathrm{p}$.

4. idem note 3

5. idem note 3

6. Attention, si horizon il y a, celui-ci est 2,6 fois plus petit que la zone noire qui se trouve au centre de cette image.

7. Il existe plusieurs alternatives théoriques aux trous noirs, capables de produire les mêmes effets sur un disque d'accrétion. Event Horizon Telescope collaboration. First M87 Event Horizon Telescope results IV. Physical origin of the asymetric ring, The Astrophysical Journal Letters, vol. $875, \mathrm{n}^{\circ} 1,2019,31 \mathrm{p}$.

8. idem note 7

9. Observatoire Européen Austral : www.eso.org

10. National Aeronautics and Space Administration : www.nasa.gov

11. Les images produites par l'ESO ou la Nasa sont proposées en libre accès sur leurs sites internet et leur utilisation est autorisée sous réserve d'indiquer les crédits.

12. Collaboration Event Horizon Telescope : www.eventhorizontelescope.org 


\section{RÉSUMÉS}

Représenter ce qui est à peine perceptible. En attendant des avancées scientifiques majeures permettant de visualiser concrètement un trou noir et pour faciliter leur vulgarisation auprès des publics non-spécialistes, des images de médiation bien différentes des représentations utilisées en astrophysique ont été créées.

\section{INDEX}

Mots-clés : médiation, outil pédagogique, patrimoine et/ou collection astronomiques, planétarium

\section{AUTEUR}

\section{MARION PARISIS}

Diplômée d'un Master Stratégie du Développement Culturel, mention Médiations de la Culture et des Patrimoines de l'université d'Avignon

marionparisis2@gmail.com 